\section{Cureus}

Received 02/22/2013

Review began 02/22/2013

Published 04/25/2013

C) Copyright 2013

Ludwig et al. This is an open access article distributed under the terms of the Creative Commons Attribution License CC-BY 3.0., which permits unrestricted use, distribution, and reproduction in any medium, provided the original author and source are credited.

\title{
From the Patient's Point of View: Patient- Centered Outcomes in Spine Surgery
}

Cassie A. Ludwig ${ }^{1}$, Anand Veeravagu ${ }^{2}$, Michael Zhang ${ }^{2}$, Mitchell Gil. Maltenfort ${ }^{3}$, Phillip Dowzicky $^{4}$, John K. Ratliff ${ }^{2}$

1. Stanford University School of Medicine 2. Department of Neurosurgery, Stanford University School of Medicine 3. Rothman Institute, Philadelphia, PA 4. University of Pennsylvania School of Medicine, Philadelphia, PA

$\square$ Corresponding author: Cassie A. Ludwig, caludwig@stanford.edu Disclosures can be found in Additional Information at the end of the article

\section{Abstract}

Introduction: A patient-centered approach to outcomes assessment necessitates knowledge of the outcomes that patients deem most important for their quality of life and overall health. To assess patient expectations with regard to seeking spine care, we conducted a prospective study of what outcome metrics were deemed most valuable to patients seeking evaluation at a tertiary spine surgery center.

Materials \& Methods: Patients seeking evaluation at an academic spine surgery practice at Thomas Jefferson University in Philadelphia were offered a survey at intake. The two-question survey explored the issues most important for consulting a neurosurgeon. Survey data was acquired over three months in early 2010. Of the surveys distributed, 335 responded, with 147 patients completing the full survey and 188 responding to the first question alone.

Results: Pain was the primary impetus for patients seeking evaluation: $73.4 \%$ of the total surveyed group reported that pain in the back or neck was one of the major concerns for their visit. Similarly, 51\% of responders indicated that pain in the back or neck was the single most important issue they wanted addressed. Frequency of responses dropped significantly for the next responses, including weakness/numbness in the leg (39.7\%), problems walking (39.7\%), pain shooting down the leg (30.7\%), and problems sleeping (30.1\%).

Conclusions: It is possible that current outcomes measures for spine surgery, including the VAS, ODI, SF-36, and EQ-5D, are either excessive or insufficient and should be adjusted to reflect patient concerns. Changing these measures, enforcing conformance with patientcentered choices of which outcome measures are most valuable, would be one approach to producing patient-specific outcomes measures in spine surgery. Alternatively, present measures may be more comprehensive, and by reducing measures to what patients care about, the quality of data captured may be limited. To determine future directions for patientcentered care, similar surveys should be rigorously conducted, reviewed, and compared to validated outcomes.

Categories: Miscellaneous, Neurosurgery

Keywords: Outcomes, practice science, quality, registry, value

\section{Introduction}

Efficient and affordable access to medical knowledge is now a reality for physicians and patients 
alike [1]. The emergence of electronic medical records (EMR) and the ubiquity and power of computing have increased the prospects for mass collection and analysis of healthcare data. Computer database systems have been established within institutions for the efficient accumulation and translation of patient data into meaningful statistics that impact the process of patient care and clinical decision-making [2]. The World Wide Web increasingly provides patients with affordable access to medical knowledge, though often of variable quality and navigability.

With a myriad of healthcare data readily available for both patients and physicians, physicians remain responsible for the meaningful synthesis and interpretation of patient data. Recent interest in patient centered outcomes and a patient-centric approach to outcomes assessment suggests that the flow of data compilation and interpretation may be reversed.

Rather than attempting to derive meaning from broadly collected outcomes data and widerange outcome metrics, physicians may begin an approach to outcomes assessment by determining what outcomes are most important to their individual patients. Data can then be centralized according to the outcomes that patients deem most important for their quality of life and overall health.

While numerous outcome measures have been reported in the literature, minimal effort has been directed at determining whether or not these measures actually capture the specific outcome metrics most valuable to patients. To begin assessing patient expectations with regard to seeking spine care, we conducted a prospective study of what outcome metrics were deemed most valuable to patients seeking evaluation at a tertiary spine surgery center.

\section{Abbreviations}

AAOS = American Academy of Orthopaedic Surgeons

$\mathrm{ACA}=$ Patient Protection and Affordable Care Act

ACO = Accountable Care Organizations

CDOSD $=$ Chronic Disabling Occupational Spinal Disorders

EMR $=$ electronic medical records

EQ-5D = EuroQOL-5D

ICCER = Institute for Comparative Clinical Effectiveness Research

ODI = Oswestry Disability Index

PCORI = Patient-Centered Research Outcomes Institute

SF-36 $=$ Short Form Health Survey

VAS = Visual Analogue Scale of Spinal Pain

VSC $=$ Vanderbilt Spine Center 


\section{Materials And Methods}

Population

This study accrued patients from an academic spine surgery practice at Thomas Jefferson University in Philadelphia. The survey was offered to all patients in intake, irrespective of previous diagnoses, therapy, or stage. There were no negative sequelae for not filling out the survey and no incentives for filling out the survey. Survey data was acquired over three months in early 2010. Of the surveys distributed, 335 responded, with 147 patients completing the full survey and 188 responding to the first question alone. The Institutional Review Board of Thomas Jefferson University reviewed the experimental protocol and provided appropriate sanction for performing the study. Informed consent was waived. The investigators complied with ethical guidelines for research involving human subjects.

\section{Measures}

The researchers developed a list of separate elements from outcome metrics widely used in spine surgery. These elements comprise the foundation measures used in compiling the Visual Analogue Scale of Spinal Pain (VAS), Oswestry Disability Index (ODI), Short Form Health Survey (SF-36), and EuroQoL Five Dimension questionnaire (EQ-5D). These common measures are widely reported in the literature for the evaluation of spine patients and have been validated in a variety of spine surgery conditions.

The two-question survey explored the issues most important for consulting a neurosurgeon (Table 1). The first question prompted respondents to "circle the top five concerns and/or complaints that you would like addressed through your visit to our clinic." The second question asked patients to "pick the single most important issue you would like addressed and underline it." 


\section{Cureus}

1) Please circle the top 5 concerns and/or complaints that you would like addressed through your visit to our clinic

2) From these, pick the single most important issue you would like addressed and underline it

Pain in my back/neck

Pain shooting down my leg

Weakness/Numbness in my leg

Amount of pain medications I am taking

Overall General Health

Problems with personal grooming/hygiene

Problems with dressing myself

Putting on shoes and socks

Problems lifting light objects

Problems lifting heavy objects

Problems walking (distance, speed)

Problems sitting

Problems standing

Problems sleeping

Problems with sex life
Limitations with traveling

Problems participating in sports

Problems climbing stairs

Problems bending over

Problems doing normal housework

(making bed, washing dishes, vacuuming)

Problems doing my job due to pain

Relationships with

co-workers/supervisor/family/friends

due to my pain

Depression or anxiety

Irritability or becoming easily angered

Feeling exhausted or tired

Problems with normal social activities

Problems with bladder control

\section{TABLE 1: Questions asked and possible responses in survey offered to patients in} intake.

\section{Statistical analysis}

Categorical data were summarized with frequency/percentage and compared.

\section{Results}

Initial survey of a patient's motivations for consulting a neurosurgeon elicits a distribution of concerns with moderate consensus (Figure 1). Pain was the primary impetus for seeking evaluation: $73.4 \%$ of the total surveyed group reported that pain in the back or neck was one of the major concerns for their visit (Table 2). Similarly, this was the primary concern affecting the majority of patients. Frequency of responses dropped significantly for the next responses, including weakness/numbness in the leg (39.7\%), problems walking (39.7\%), pain shooting down the leg (30.7\%), and problems sleeping (30.1\%). For the remaining options, about a quarter or less of patients indicated that one was among his or her major concerns. Sexual and social issues and return to work were of very low frequency, with such activities selected by less than $15 \%$ of patients. 


\section{Cureus}

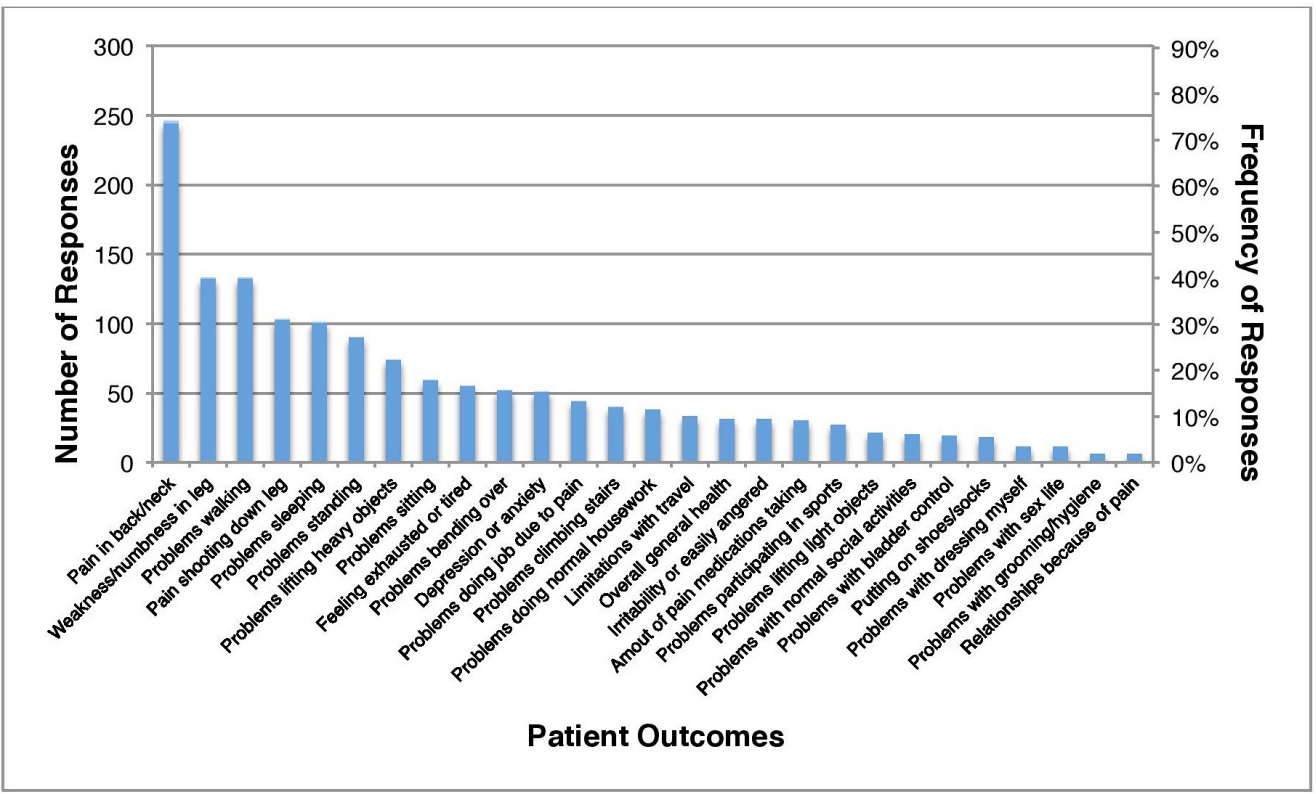

FIGURE 1: Top concerns and/or complaints patients wanted addressed during visit

\section{Outcome}

Pain in back/neck

Weakness/numbness in leg

Problems walking

Pain shooting down leg

Problems sleeping

Problems standing

Problems lifting heavy objects

Problems sitting

Feeling exhausted or tired

Problems bending over
Responses ( $\mathrm{N}=335)$

246

133

133

103

101

90

74

59

55

52
Frequency (\%)

30.7

30.1

26.9

22.1

17.6

16.4

15.5

TABLE 2: Most common responses selected by patients when asked to indicate top five concerns and/or complaints to be addressed. 


\section{Cureus}

responses were similar (Figure 2). As before, pain in the back or neck was a dominating issue as indicated by $51 \%$ of responders (Table 3). Weakness/numbness in the leg (13.6\%), pain shooting down the leg (6.1\%), and problems with walking (5.4\%) contributed to the top four issues. These last three lower limb concerns compose approximately one-quarter (25.1\%) of the available responses. None of the remaining individual options assumed more than $5 \%$ of the total responding sample.

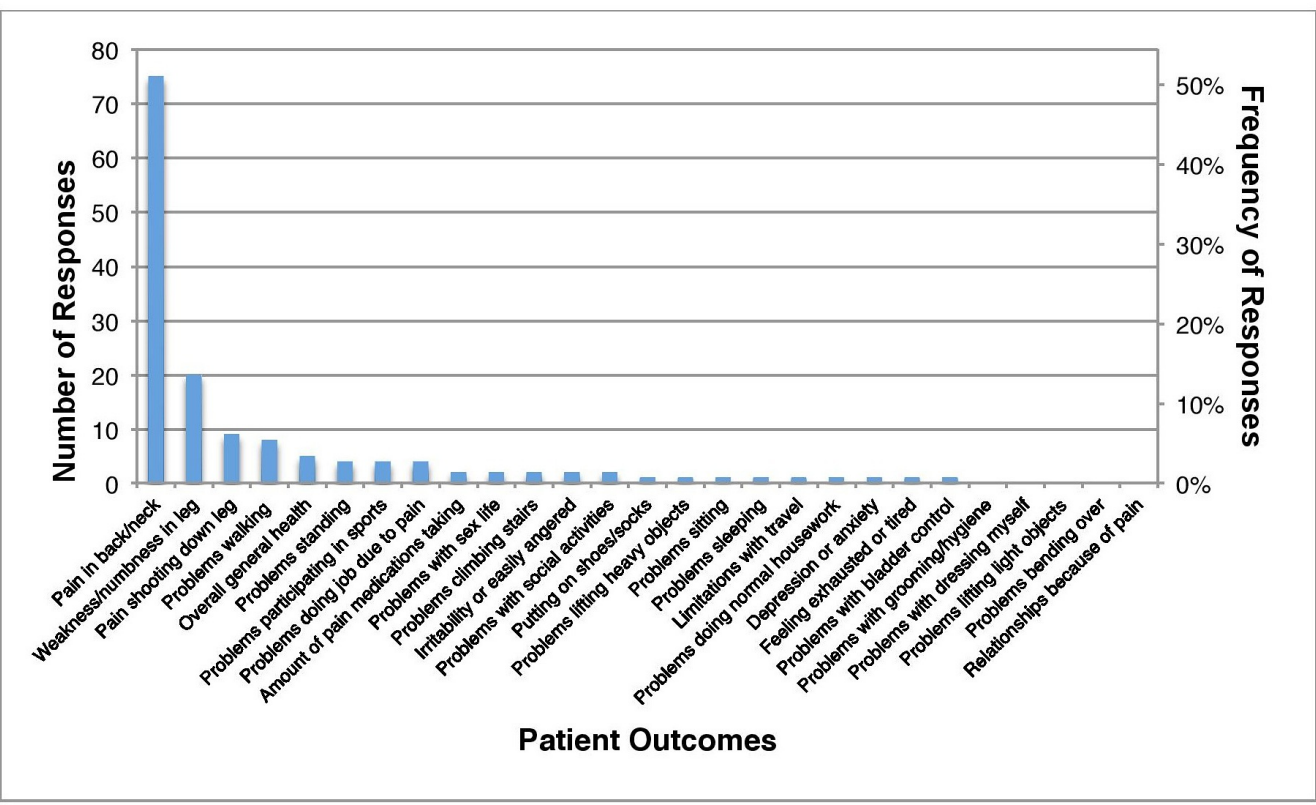

FIGURE 2: Single most important issue 


\section{Cureus}

Outcome

Pain in back/neck

Weakness/numbness in leg

Pain shooting down leg

Problems walking

Overall general health

Problems standing

Problems participating in sports

Problems doing job due to pain

Amount of pain medications taking

Problems with sex life

\section{Frequency (\%)}

51

6.1

5.4

3.4

2.7

2.7

2.7

1.4

1.4

\section{TABLE 3: Most common responses selected by patients when asked to indicate}

single most important issues for consulting neurosurgeon.

Pain was consistently the most common complaint voiced by patients. Axial neck or back pain was the most common complaint offered for both the top five issue and single issue questions. Radicular pain was the third most common single complaint and fourth most common complaint from the multiple issue question.

Job problems were not a common complaint for this cohort of patients. Only $13.1 \%$ of patients noted that problems doing their job due to pain were among the top five issues prompting evaluation at a spine center. Only $2.7 \%$ noted this was the most important issue for which they sought evaluation.

Notably, only $43.8 \%$ of the total patient sample ultimately provided a submission for the survey's second question. The implications of this partial response rate are uncertain and varied, as this could reflect a patient's unwillingness or inability to provide a single answer, or could represent a deficiency in survey design or execution. While the number of responses tallied for the single most important issues are deflated, the true percentage distribution cannot be determined.

\section{Discussion}

\section{Patient-centered outcomes}

The results of this study indicate that pain in the back or neck is the single most important issue that prompts a patient to consult a spine surgeon. This may suggest higher utility for pain-centered validated outcomes measures, such as the VAS. Pain is included in the SF-36, EQ-5D and ODI as well, though it is only one of eight dimensions that contribute to the total score for the SF-36, only one of five for the EQ-5D, and distributed throughout the sections of the ODI. In the results of both survey questions, the three concerns that followed pain in the 
back or neck in importance to patients involved both pain and the functional consequences of their pathology: weakness/numbness in the leg, pain shooting down the leg, and problems with walking. Only walking is addressed in the SF-36 and EQ-5D, while all these specific measurements are incorporated within the ODI.

It is possible that other outcomes measured by spine surgeons (ex: self-care, daily activities, and anxiety/depression) are not patient-centered as the majority of patients did not indicate that these issues were of primary concern. Therefore, this survey raises the question of whether neurosurgical outcomes assessments should be moved closer to assessments of the issues identified as most important to patients. This may enable physicians to quantify patient expectations without relying on previously validated outcomes measurements that over- or underemphasize concerns.

\section{The Patient Protection and Affordable Care Act}

The Patient Protection and Affordable Care Act (ACA) was signed into law March 23, 2010 and will be fully implemented on January 1, 2014 [3]. Since the creation of the ACA, the emphasis of health care reform has shifted considerably from quantity to quality [4-7]. Present efforts seek to link physician reimbursement to "value measures" which take into account both quality and cost variation [8]. ACA legislation will invest nearly \$1.1 trillion between 2012 and 2021 in an attempt to make coverage affordable-an investment hoped to be offset in part by a reduction in Medicare and Medicaid spending through an emphasis on high quality and low cost care [9]. Therefore, data on effectiveness of care in everyday practice will be imperative to ensure that patients receive the highest quality rather than simply the lowest-cost care in the future.

The ACA introduces several changes pertinent to the present shift in focus on quality of care, three of which include the creation of Accountable Care Organizations (ACOs), a National Quality Strategy, and the Institute for Comparative Clinical Effectiveness Research. The first, ACOs, include a group of physicians and hospitals that will take responsibility for an expressed population of patients [10]. ACOs will provide physicians and hospitals with incentive to improve integration and increase quality measurement and reporting, ultimately leading to improved outcomes and a reduction in healthcare cost. A central measurement of performance in ACOs is the patient-reported outcome, which further incentivizes physicians to focus patient care and quality measures on patient-specific concerns.

The second, the development of a multi-payer National Quality Strategy, aims to generate multi-payer quality and efficiency measures to promote value purchasing, improve safety, and provide increased health information to private and public insurers [3]. In addition to the drive for increased comparative effectiveness research, The National Quality Strategy encourages increased performance reporting to increase patient's access to information about their care and about the quality of their care.

Lastly, the Institute for Comparative Clinical Effectiveness Research (ICCER) was established by the ACA "to assist consumers, clinicians, purchasers, and policy makers to make informed decisions that improve health care at both the individual and population levels" [3, 11]. This builds on the American Recovery and Reinvestment Act of 2009 that allocated 1.1 billion dollars for new comparative effectiveness research in the U.S [12].

\section{Patient-Centered Outcomes Research Institute}

The Patient-Centered Research Outcomes Institute (PCORI) was created as part of the ACA effort to foster research on informed decision-making, patient care, and re-directing research efforts to be patient-centered. The PCORI has led to an increased focus on patient-centered outcome measures and on framing research in a fashion immediately applicable to patients. 


\section{Cureus}

The foundation of the PCORI approach may ultimately move outcomes assessment away from previously validated outcome measures. While many outcomes projects have been completed in spine surgery, limited research has been directed at understanding patient expectations and ensuring that outcome metrics appropriately capture the goals of patients in seeking care.

\section{Outcome metrics in spine surgery}

Spine surgery serves as an appropriate model for patient centered outcomes in neurosurgery as it is an elective procedure performed for a degenerative process. Patient outcomes are therefore of utmost importance in a patient's decision to proceed with surgery.

Current validated measures for patient outcomes in spine surgery include the VAS [13], ODI [14-16], SF-36 [17], and EQ-5D [18-20] (Table 4). The VAS is a self-reported, one-dimensional pain rating scale. In contrast to the VAS, the ODI is a self-reported questionnaire with seven (AAOS version 1.0) sections. The ODI measures condition-specific disability and focuses on pain and can be used for both assessment and outcomes data collection. A more general measure of quality of life, the SF-36, is a multipurpose, short-form health survey with 36 items that yields an eight-scale profile of scores as well as overall physical and mental health summary measures [17]. Similarly, the EQ-5D is a two-part self-reported multi-dimensional questionnaire that generates 243 possible health states based on three possible answers for five dimensions [19]. The EQ-5D also measures general quality of life and yields a single utility score of 0-1:0 representing death and one representing full health.

\begin{tabular}{|c|c|c|c|c|}
\hline & VAS & ODI & SF-36 & EQ-5D \\
\hline Pain & Pain & * & Bodily Pain & Pain/Discomfort \\
\hline Functionality & & $\begin{array}{l}\text { Walking, Sitting, Standing, } \\
\text { Sleeping, Lifting, Traveling, } \\
\text { Personal Care }\end{array}$ & $\begin{array}{l}\text { Physical Functioning, Role- } \\
\text { Physical, General Health, Social } \\
\text { Functioning, }\end{array}$ & $\begin{array}{l}\text { Mobility, Daily } \\
\text { Activities, Self- } \\
\text { Care }\end{array}$ \\
\hline Mental Health & & & $\begin{array}{l}\text { Role-Emotional, Mental Health, } \\
\text { Vitality }\end{array}$ & Anxiety/Depression \\
\hline
\end{tabular}

\section{TABLE 4: Validated measures for patient outcomes in spine surgery}

* Incorporated throughout the Measure

These measures are frequently used in conjunction to report patient outcomes pre- and postsurgery. However, they may not all represent the outcomes most important to patients themselves.

\section{Study limitations}

The external validity of the study is limited by the fact that it is a single-institution study with a small cohort. Limiting the study to a single institution yields optimal data for the institution itself. However, to obtain more generalizable data, future multi-institutional studies should be performed. Additionally, the generalizability of this study is limited by the high prevalence of psychiatric disorders amongst spine patients. Dersh et al. have reported that a majority (65\%) of patients with chronic disabling occupational spinal disorders (CDOSD) were diagnosed with at least one current disorder (not including Pain Disorder), compared with only $15 \%$ of the 
general population [21]. While the patient population in this study is composed of all patients at an academic spine surgery practice with no inclusion criteria based on diagnosis, the potential increase in psychiatric disorders amongst this population must be considered as a variable affecting patient centered outcomes.

Furthermore, lack of an overall calculated response-rate yields high response bias and a lower response-rate for question two [188/335 (56.1\%)] limits the internal validity of this study. A more rigorous approach to survey administration and collection should be applied in future studies to maximize internal and external validity and minimize response bias.

Collecting both admission and follow-up data may yield more information as to the benefits/constraints of patient-centered outcomes in the future. To obtain this data, the stringent methodology utilized by Berkowitz et al. in developing a Gamma Knife radiosurgery registry could be applied [2]. Berkowitz et al. ensure 100\% patient inclusion in studies by monitoring their 11,738 member patient registry through the use of clerical staff checks, server data back up, and quality assessment in six-month intervals via built-in software. Similarly, the Vanderbilt Spine Center (VSC) includes all patients undergoing spine surgery in a prospective longitudinal spine registry, regardless of diagnosis or surgical treatment through the use of registry associates, Web-based Research Electronic Data Capture, and EMR [22]. The VSC employs validated outcomes measures to capture baseline, three month and 12 month outcomes data. Such strict forms of data-collection will be critical in developing accurate data to provide true patient-centered outcomes on an institution basis in the future. Cost and infrastructure development remain the largest drawbacks to this methodology.

\section{Results of this study}

Knowing the issues of greatest concern to patients seeking evaluation for spinal disease will provide clinicians with the opportunity to reassess measurements of surgery outcomes. Assessment of patient expectations in seeking spine care may offer a valuable means to ensure that patients have appropriate expectations in seeking surgical treatment.

In this survey, the majority of patients seeking evaluation at a spine surgery center did so for complaints of pain. Some concerns that are focused upon by outcome metrics used in spine surgery were not listed as primary concerns by patients. Functional deficits, as outlined by the SF-36 measure, were not commonly noted to be primary issues for which individual patients sought treatment. Similarly, return to work is often reported as a proxy for recovery after spine surgery procedures. However, this patient cohort did not note that workplace concerns were significant to their seeking evaluation. This may, in part, explain the results of a study by Hodges et al. that found no correlation between OSW, VAS and SF-36 and return-to-work rates in 87 workers' compensation patients who underwent lumbar spine surgery [23].

Present approaches to outcome measures in spine surgery may not be entirely representative of patient expectations. Reviewing the commonly used outcome measures and comparing them to patient choices for most important issues for consulting a spine surgeon, the VAS is the single most valuable outcome metric. The ODI, similarly through relating pain to functional level, captures many issues deemed important by patients.

\section{Conclusions}

It is possible that measures used today are insufficient and should be adjusted to reflect patient concerns. Some present, widely-used outcomes measures report elements that patients may not find valuable. Changing these measures, enforcing conformance with patient-centered choices of which outcome measures are most valuable, would be one approach to producing patient-specific outcomes measures in spine surgery. 
Alternatively, present measures may be more comprehensive, and by reducing measures to what patients care about, the quality of data captured may be limited. Routinely used and validated outcome measures may be providing a broader measure of patient outcomes. By focusing solely on what patients are interested in, physicians may be limiting their opportunity to improve patient health across multiple measures.

Both possibilities have implications for the future of patient-centered care in neurosurgery. As healthcare transforms under reform efforts, it is important for physicians and patients alike to recognize that what makes patients happier does not necessarily make them healthier. The goals of health care need to be defined on a national, institutional and individual basis.

To determine future directions for patient-centered care, similar surveys should be rigorously conducted, reviewed, and compared to validated outcomes. A survey of both patients and surgeons should be performed to determine whether patients and health professionals differ in their perspectives with respect to quality in spine surgery.

\section{Additional Information}

\section{Disclosures}

Human subjects: Consent was obtained by all participants in this study. The Institutional Review Boards of Thomas Jefferson University issued approval N/A. Animal subjects: All authors have confirmed that this study did not involve animal subjects or tissue. Conflicts of interest: In compliance with the ICMJE uniform disclosure form, all authors declare the following: Payment/services info: All authors have declared that no financial support was received from any organization for the submitted work. Financial relationships: All authors have declared that they have no financial relationships at present or within the previous three years with any organizations that might have an interest in the submitted work. Other relationships: All authors have declared that there are no other relationships or activities that could appear to have influenced the submitted work.

\section{Acknowledgements}

This article has not been submitted for publication elsewhere, nor has it been published elsewhere in whole or in part. We authors report no conflicts of interest, and have received no funding for this project. This study was performed with the approval of the Institutional Review Board of Thomas Jefferson University. Informed consent was waived. The investigators complied with ethical guidelines for research involving human subjects. All authors listed on the title page have read and substantially contributed to the writing of the manuscript. No other persons have contributed significantly to the manuscript's preparation. This information was added on 3/8/14.

\section{References}

1. Asher AL, McCormick PC, Kondziolka D: Introduction: The science of practice: addressing the challenges of modern health care. Neurosurgical Focus. 2013, 34:Introduction.

2. Berkowitz O, Kondziolka D, Bissonette D, Niranjan A, Kano H, Lunsford LD: The evolution of a clinical registry during 25 years of experience with Gamma Knife radiosurgery in Pittsburgh. Neurosurgical Focus. 2013, 34:E4. 10.3171/2012.11.FOCUS12295

3. The Patient Protection and Affordable Care Act (PPACA), Law P No. 111-148, 124 Stat. 119 . (March 23, 2010).

4. Rosenbaum S: The Patient Protection and Affordable Care Act: implications for public health policy and practice. Public health reports (Washington, D.C.: 1974. 2011, 126:130-135.

5. Fisher ES, McClellan MB, Safran DG: Building the Path to Accountable Care. NEJM. 2011, 365:2445-2447. 
6. McWilliams JM, Song Z: Implications for ACOs of variations in spending growth. NEJM. 2012, 366:e29.

7. Roder C, Staub L, Dietrich D, Zweig T, Melloh M, Aebi M : Benchmarking with Spine Tango: potentials and pitfalls. European spine journal: official publication of the European Spine Society, the European Spinal Deformity Society, and the European Section of the Cervical Spine Research Society. 2009, 18:305-311.

8. McGirt MJ, Speroff T, Dittus RS, Harrell FE, Asher AL: The National Neurosurgery Quality and Outcomes Database (N2QOD): general overview and pilot-year project description. Neurosurgical Focus. 2013, 34:E6.

9. Updated Estimates for the Coverage Provisions of the Affordable Care Act . ( March 2012). Accessed: January 2013: http://www.cbo.gov/.

10. Berwick DM: Making good on ACOs' promise--the final rule for the Medicare shared savings program. NEJM. 2011, 365:1753-1756.

11. Initial National Priorities for Comparative Effectiveness Research. Washington, DC: The National Academies Press; IOM (Institute of Medicine). (2009).

12. American Recovery and Reinvestment Act of 2009, Law P No. 111-5, H.R. 1 Stat. 119 . (February 17, 2009).

13. Gallagher EJ, Liebman M, Bijur PE: Prospective validation of clinically important changes in pain severity measured on a visual analog scale. Annals of Emergency Medicine . 2001, 38:633-638.

14. Fairbank JC, Couper J, Davies JB, O'Brien JP: The Oswestry low back pain disability questionnaire. . Physiotherapy . 1980, 66:271-273.

15. Fairbank JC, Pynsent PB: The Oswestry Disability Index. Spine. 2000, 25:2940-2952.

16. Roland M, Fairbank J: The Roland-Morris Disability Questionnaire and the Oswestry Disability Questionnaire. Spine. 2000, 25:3115-3124.

17. Ware JE, Jr.: SF-36 health survey update. Spine . 2000, 25:3130-3139.

18. Adogwa O, Parker SL, Shau D, Mendelhall SK, Cheng J, Aaronson O, Devin CJ, McGirt MJ: Long-term outcomes of revision fusion for lumbar pseudarthrosis: clinical article . Journal of Neurosurgery. Spine. 2011, 15:393-398.

19. Jansson KA, Nemeth G, Granath F, Jonsson B, Blomqvist P: Health-related quality of life (EQ$5 \mathrm{D})$ before and one year after surgery for lumbar spinal stenosis. The Journal of Bone and Joint Surgery. British volume 2009, 91:210-216.

20. Parker SL, Adogwa O, Paul AR, Anderson WN, Aaronson O, Cheng JS, McGirt MJ: Utility of minimum clinically important difference in assessing pain, disability, and health state after transforaminal lumbar interbody fusion for degenerative lumbar spondylolisthesis. Journal of Neurosurgery. Spine . 2011, 14:598-604.

21. Dersh J, Gatchel RJ, Mayer T, Polatin P, Temple OR: Prevalence of psychiatric disorders in patients with chronic disabling occupational spinal disorders. Spine. 2006, 31:1156-1162.

22. McGirt MJ, Speroff T, Godil SS, Cheng JS, Selden NR, Asher AL: Outcome science in practice: an overview and initial experience at the Vanderbilt Spine Center. Neurosurg Focus. 2013, 34:E7.

23. Hodges SD, Humphreys SC, Eck JC, Covington LA, Harrom H: Predicting factors of successful recovery from lumbar spine surgery among workers' compensation patients. J Am Osteopath. 2011, 101:78-83. 